\title{
Multiple Features Fusion System for Motion Recognition
}

\author{
Jiang Hua ${ }^{1,2}$, Zhaojie $\mathrm{Ju}^{3}$, Disi Chen ${ }^{3}$, Dalin Zhou ${ }^{3}$, Haoyi Zhao ${ }^{4}$, Du Jiang ${ }^{5,6}$ and \\ Gongfa $\mathrm{Li}^{1,2 \text {, * }}$ \\ ${ }^{1}$ Key Laboratory of Metallurgical Equipment and Control Technology of Ministry of \\ Education, Wuhan University of Science and Technology, Wuhan 430081, China \\ ${ }^{2}$ Precision Manufacturing Research Institute, Wuhan University of Science and Technology, \\ Wuhan 430081, China \\ ${ }^{3}$ School of Computing, University of Portsmouth, Portsmouth PO1 3HE, UK \\ ${ }^{4}$ Research Center of Biologic Manipulator and Intelligent Measurement and Control, Wuhan \\ University of Science and Technology, Wuhan 430081, China \\ ${ }^{5}$ Hubei Key Laboratory of Mechanical Transmission and Manufacturing Engineering, Wuhan \\ University of Science and Technology, Wuhan 430081, China \\ ${ }^{6}$ 3D Printing and Intelligent Manufacturing Engineering Institute, Wuhan University of \\ Science and Technology \\ * ligongfa@wust.edu.cn
}

\begin{abstract}
Surface EMG signal is a signal source that can reflect the movement state of human muscles accurately. However, there are still problems such as low recognition rate in practical applications. It is necessary to study how they can be exploited effectively for a more accurate extraction. The paper combines two time domain features and nonlinear feature to get the feature vector for subsequent pattern recognition. The paper chooses the generalized regression neural network (GRNN) as the classifier for hand motion pattern recognition. The proposed method in this paper not only realizes the feature extraction of signals, but also ensures the high classification accuracy. The feature, RMS-SampEnWL, obtains the highest recognition rate above 97\% compared with the two time features. The new sEMG feature is effective and suitable for hand motion pattern recognition. Finally, we hope to establish a robust recognition system based on sEMG.
\end{abstract}

Keywords: Surface EMG signal, GRNN classifier, RMS-SampEn-WL.

\section{Introduction}

The pattern recognition technology used in surface electromyography (sEMG) signals for identifying human motions is widely involved in human-computer interaction, biomimetic prosthetics, diagnosis in clinical applications and the development of rehabilitation equipment [1].

The research is more challenging compared to other widely used bioelectrical signals because of its randomness [2]. sEMG is a biological signal that is collected on the surface of the skin [3]. The muscle control information can be collected to control 
electrical powered prostheses [4]. So this method based on sEMG Signal is widely used in a series of clinical applications [5].

Therefore, many researchers have begun to study the features of the sEMG signals. They have re-evaluated the performance of the commonly used Hudgins' time domain features [6]. Autoregressive (AR) model analysis is also a common method to get signal feature [7]. Willison amplitude(WAMP) and sample entropy (SampEn) using six different sEMG data sets containing over 60 subject sessions and 2500 separate contractions [8]. In addition, multiple features are studied or evaluated to improve the hand motion classification accuracy [9]. A set of time domain features is proposed that can estimate the EMG signal power spectrum characteristics using five different EMG data sets [10].

The current research on the physiological mechanism of human motion is still in the exploratory stage [11]. Therefore, in the process of pattern recognition, it is still lack of a standard in these aspects: selection of the sampled muscle groups, the extraction of sEMG features, and the modeling of continuous motions [12]. Universally, researchers determine the program mainly based on previous experience, these subjective factors may bring more uncertainty into the experimental conclusions [13].

Obtaining the active segment data of continuous sEMG signals accurately is the primary procedure to complete the classification of action patterns [14]. Especially in the mode of supervised machine learning, a sufficient data samples of different action types is the premise and guarantee of the classifier with excellent performance.

The purpose of the pattern classification is to determine which model the features fit. A good pattern classifier should have a smaller amount of computation, a higher recognition rate, and a stronger generalization ability. Support vector machine (SVM), linear discriminant analysis (LDA), k-nearest neighbor (KNN), multi-layer perceptron neuron network (MLP) and random forest (RF) classification algorithm are put forward [15].

In view of the above situations, this paper focuses on proposing an exploratory research to fuse multiple features for improving sEMG classification. We prove that the pre-processed sEMG frequency domain signals have a large loss through experimental analysis, so the time domain features are extracted for classifying. The nonlinear dynamic feature can construct a multidimensional dynamic model based on a onedimensional time series to extract more hidden information. So large amounts of experiments have been conducted to explore how features fuse can achieve a better performance. The time domain features this paper choose are root mean square (RSM) and Waveform length (WL), the nonlinear dynamic feature is sample entropy (SampEn). The experimental result shows that this new feature can improve the accuracy of different hand motions classification based on sEMG after comparing the new feature with traditional time domain features. The feature contributes to the accurate human-machine interaction between intelligent prosthetic hand and users.

The remainder of this paper is organized as follows. Section 2 describes the methods to capture and process sEMG signals. Section 3 indicates the experimental results about the feature extraction and performance evaluation. Section 4 draws the conclusion and gives the discussion of future work. 


\section{Related work}

\subsection{Collection of signals}

We collect the sEMG signals of nine hand motions from 10 subjects. All of them were healthy able-bodied subjects, without any prior neuromuscular disorders.

sEMG is usually collected by one or more electrodes were placed on the skin surface. Electrode shift and electrode distribution will obviously affect the recognition accuracy of the hand motion. Experiments use a 16-channel electrode sleeve to collect experimental data. In this paper the electrodes are modified from traditional disposable electrodes by gel removing means. Elastic fabrics are used to fix electrodes in advance. This method can reduce the time and position error of arranging electrodes. To obtain better skin impedance, the subjects must use alcohol to wash the forearm skin before the experiment. The scene of wearing the sleeve is shown in Figure 1.

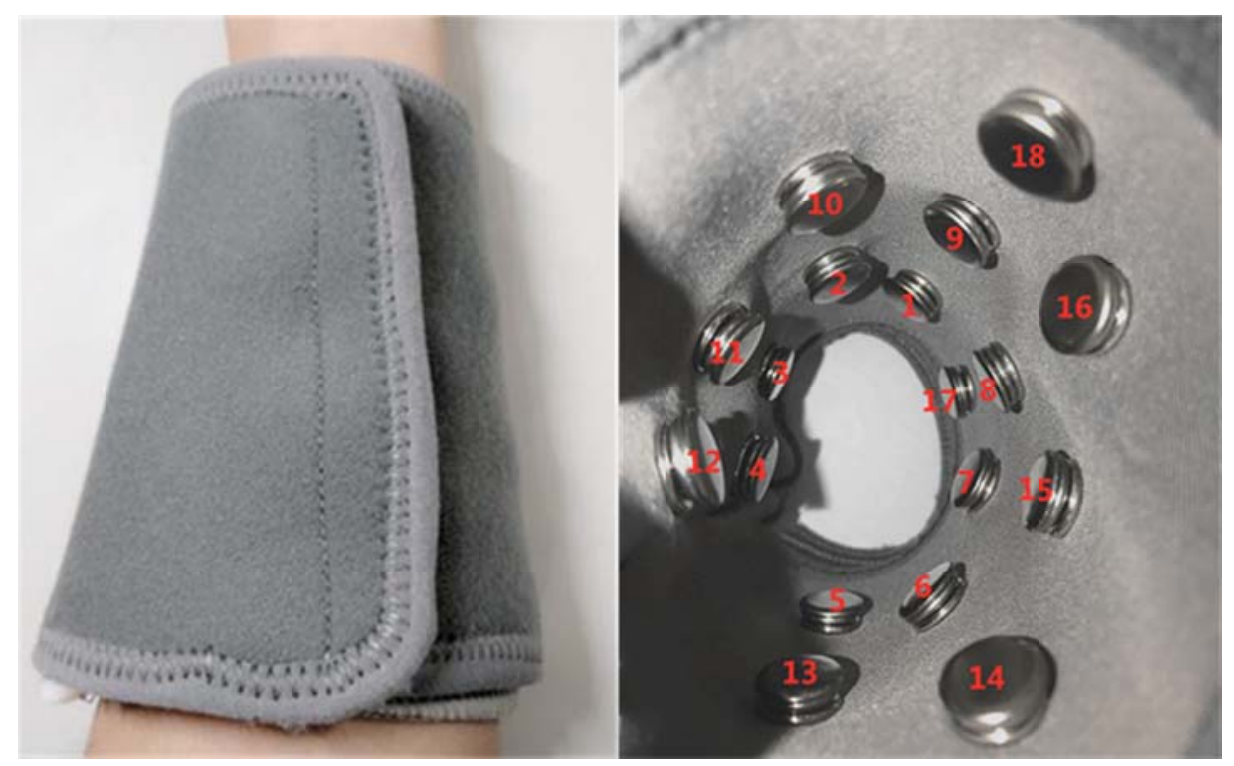

Fig. 1. It shows the scene of wearing the sleeve.

The 9 motions include hand closure (HC), hand open (HO), wrist flexion (WF), wrist extension(WE), thumb force on index finger (TI), middle finger (TM), ring finger (TR) and the little finger (TL), in addition to these hand motions also include a rest action (RE). The experimental hand motions are showed in Figure 2. 


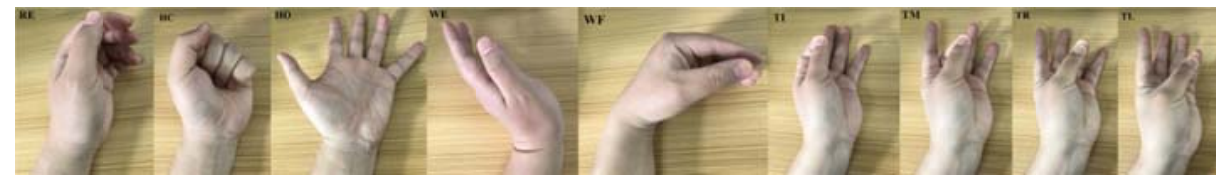

Fig. 2. The experimental hand motions

In the experiment, 10 trials were repeated for each motion. The repeated method is to rest for $5 \mathrm{~s}$, keep the action for $5 \mathrm{~s}$, repeat 10 times. If you have finished a motion, there will be a ten-minute break to avoid muscle fatigue. The hand motions are collected for 3 consecutive days, using the same collection method. So the temporal and spatial differences of the same individual in myoelectric signals can be obtained.

\section{$2.2 \quad$ Signal preprocessing}

In order to achieve a high recognition rate of the classification results, the raw SEMG signals need to be preprocessed. The EMG signals contain a variety of noises due to its instability. The preprocessing includes two steps of noise reduction and feature selection. The digital noise filtering is combined with wavelet transform to reduce the true signal waveform as much as possible.

The captured sEMG signal consists of 4 phases: The relax state of a hand, The dynamic process for forming a hand motion, the steady state for maintaining a hand motion and the dynamic procedure for releasing a hand motion. In the experiment, sEMG signals sampling frequency is set to $1 \mathrm{kHz}$ based on the Nyquist Sampling Theorem.

Power frequency interference caused by the nonlinear characteristics of hardware must be removed first. The power frequency of experimental equipment is $50 \mathrm{~Hz}$. To eliminate these interferences, the experiment uses a 20 -step $50 \mathrm{~Hz}$ comb fillter to remove power frequency noise. The comb filter results are shown in Figure 3. 

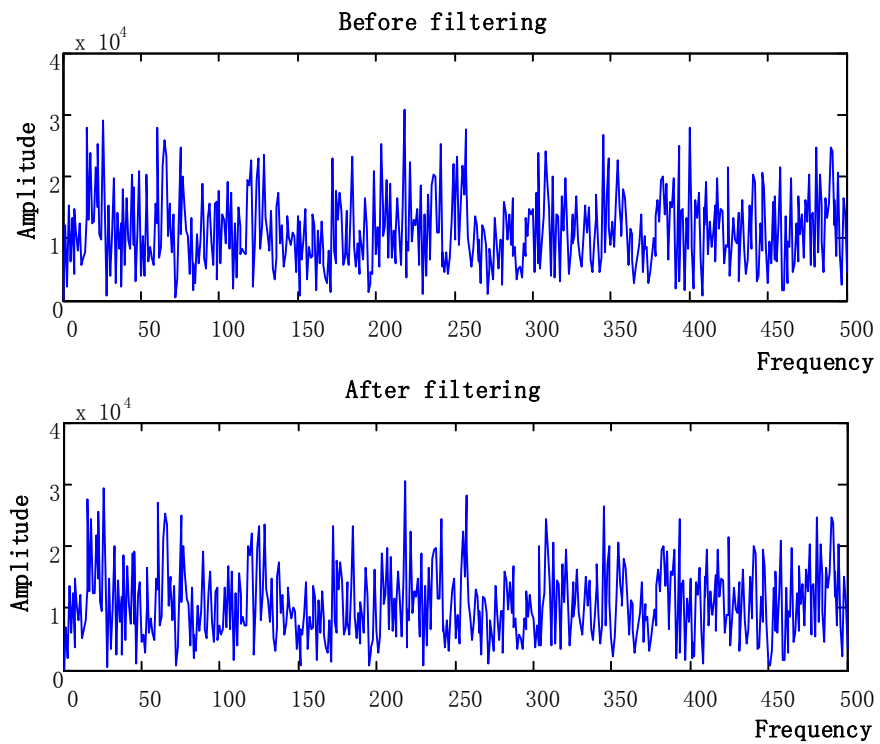

Fig. 3. Comparing the two graphs, it can be found that the filter mainly deals with the signals at an integer multiple of $50 \mathrm{~Hz}$.

The preprocessed signals still contain a large amount of data that will affect the operation speed. Therefore, the valid signals should be extracted as a feature value before the sEMG signals put into the classifier. The main function of feature extraction is to make classification easier and more intuitive by way of mapping complex signals from high-dimensional space to low-dimensional space.

Many scholars have done a lot of work on feature extraction and pattern recognition. The time domain methods treat the sEMG signals as a function of time, and obtain some features by time domain analysis. The time domain methods are widely used in feature extraction of sEMG signals because of the lower computation in algorithm complexity. However, even small changes in muscle activity will cause significant influence. The frequency domain methods of sEMG signals are relatively stable because of the signals transformed into power spectrum and the waveform change little. But the sEMG signals will have a great loss in the process of extracting features.

This paper compares two time-domain features and nonlinear feature to prove the effectiveness. Two time domain features include root mean square (RMS) and waveform length (WL).

RMS is the measure of the amplitude of the EMG signal, which can be expressed as (1).

$$
R M S=\sqrt{\frac{1}{N} \sum_{i=1}^{N} x_{i}^{2}}
$$

$\mathrm{N}$ is the length of the window. Similar to RMS, the absolute value of the integral and the mean absolute value (MAV) have been proven to have the same performance in 
manual identification.

WL refers to the cumulative length of the EMG waveform. WL is related to the waveform amplitude, frequency and time. Where $\mathrm{N}$ represents the length of the signal and $X_{n}$ represents the EMG signal in a segment. It can be formulated as (2).

$$
W L=\sum_{i=1}^{N-1}\left|x_{i+1}-x_{i}\right|
$$

Although the two methods have been widely used, they ignore the robustness of stochastic sEMG signals. The number of motor units and action potentials vary dramatically during the process of exercise, and the motion-neural system has many nonlinear characteristics. So the nonlinear dynamics methods can construct a multidimensional dynamic model to extract more hidden information. This paper chooses the main nonlinear method sample entropy (SampEn).

SampEn similar to the approximate entropy is used to measure the randomness of dynamic system expressed in time series. However, it has been used to reduce the error caused by the comparison of its own data. The equation of SampEn is defined as (3).

$$
\operatorname{SampEn}(m, r, N)=-\operatorname{In} \frac{A}{B}
$$

As is defined in (1), $r$ is the threshold generally given, $N$ is the number of samples and $m$ is a given embedding dimension.

So the sample entropy, time domain features RMS and WL are used as eigenvalues to form feature vectors.

$$
\text { feature }=\{R M S, W L, \text { SampEn }\}
$$

\section{Experimental result}

\subsection{Feature extraction}

In this paper, the EMG signals are collected simultaneously by 16 channels, so the dimension of the three features is: $1 * 48$. The mean value of the 16 channels is taken as the valid description to perform the feature vector. As shown in Table 1, the mean values of the three features under the nine motions.

Table 1. The mean values of the three features under the nine motions.

\begin{tabular}{cccc}
\hline & RMS & WL & SampEn \\
\hline $\boldsymbol{R e}$ & 7.1262 & $1.8368 * \mathrm{e} 3$ & 0.9410 \\
$\boldsymbol{H C}$ & 183.1176 & $4.3872 * \mathrm{e} 4$ & 0.9133 \\
\hline
\end{tabular}




\begin{tabular}{cccc}
\hline HO & 125.5576 & $2.5299^{*} \mathrm{e} 4$ & 1.5337 \\
WE & 42.9087 & $1.1577^{*} \mathrm{e} 4$ & 1.1249 \\
$\boldsymbol{W} \boldsymbol{F}$ & 50.0360 & 1.335 * $^{\mathrm{e}} 4$ & 1.3516 \\
$T \boldsymbol{I I}$ & 58.4510 & $9.3138^{*} \mathrm{e} 3$ & 1.1672 \\
$T M$ & 75.1499 & $1.3223^{*} \mathrm{e} 4$ & 1.4571 \\
$T \boldsymbol{R}$ & 102.8147 & $2.1140 * \mathrm{e} 4$ & 0.9705 \\
$T \boldsymbol{T}$ & 28.5878 & $6.6026 * \mathrm{e} 3$ & 1.0930 \\
\hline
\end{tabular}

It can be clearly seen that the mean values of three features extracted from the signals are significantly different. In order to further show the difference of the feature vector, this paper takes ten sample points of the action to draw a scatter plot, as shown in Figure 4.

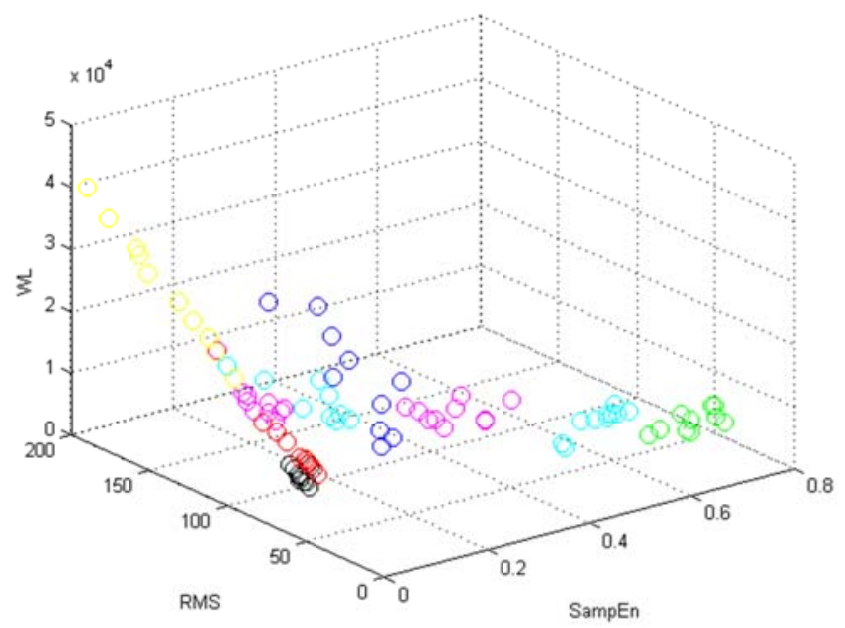

Fig. 4. A scatter plot of RMS-SampEn-WL.

It can be seen from Figure 4 that the distribution of the nine hand movements is relatively discrete, although the distribution areas of the black and red portions are close. So this part of the area is enlarged, as shown in Figure 5. 


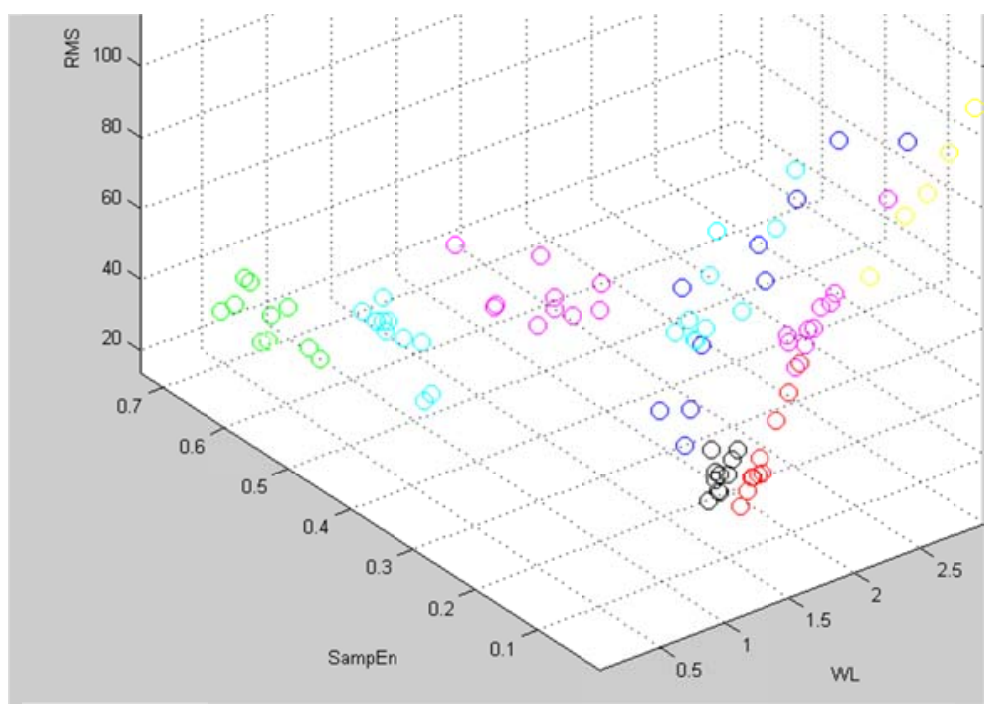

Fig. 5. The enlarged area of the black and red portions.

It can be found that the distribution area has obvious interval. Therefore, it is feasible to extract the sample entropy, RMS and WL as the features of hand motions recognition for classification and recognition research.

\subsection{Performance evaluation}

After completing the preparations such as noise reduction and feature extraction, the features need to be put into the appropriate classifier. The classifier is particularly important to realize the final hand motion classification and recognition. Currently, there are many types of widely used classifiers, and they have their own advantages and shortcomings. It is necessary to analyze the best hand motion classifiers through certain comparative experimental analysis. By summarizing and analyzing the existing work, this chapter chooses the generalized regression neural network (GRNN) as the classifier for hand motion pattern recognition.

Firstly, the feature vectors and corresponding action labels of the 9 groups of motions are equally divided into two groups, which are used as the training data set and the test data set respectively. At the same time, the data will be normalized. The data scaling involved in this paper mainly refers to scale the feature value WL to avoid the excessively dominant feature vector. After the parameters are optimized, the scaled training data set is imported into the GRNN classifier.

This paper compares the fusion feature with two time features to prove the effectiveness of the proposed method. The test data set is put into the classifier model to complete the test, and the GRNN classification performance is shown in Figure 6 to Figure 8. 
The classification result of RMS

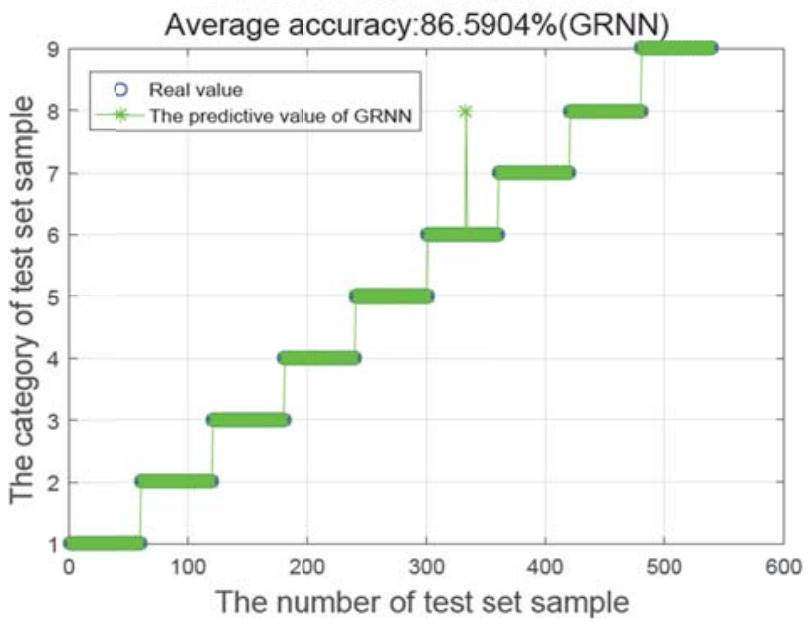

Fig. 6. The classification result of RMS.

The classification result of WL Average accuracy:89.1204\%(GRNN)

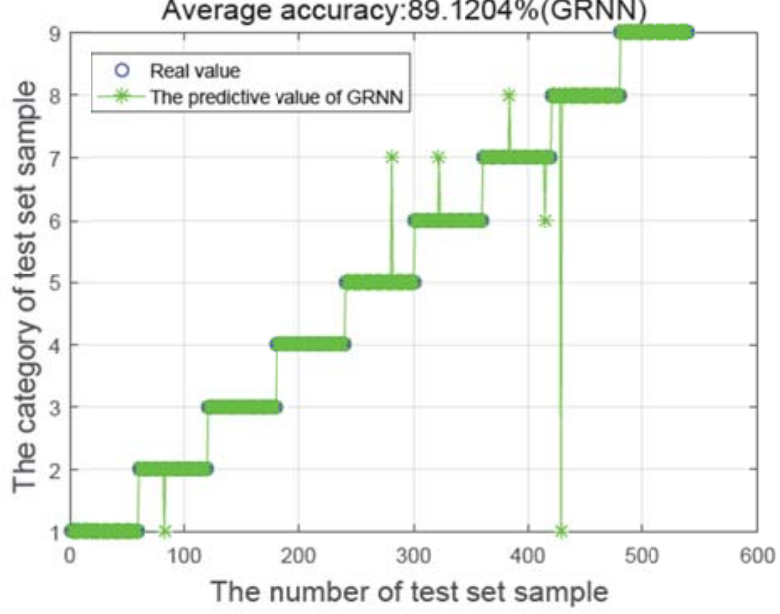

Fig. 7. The classification result of WL. 


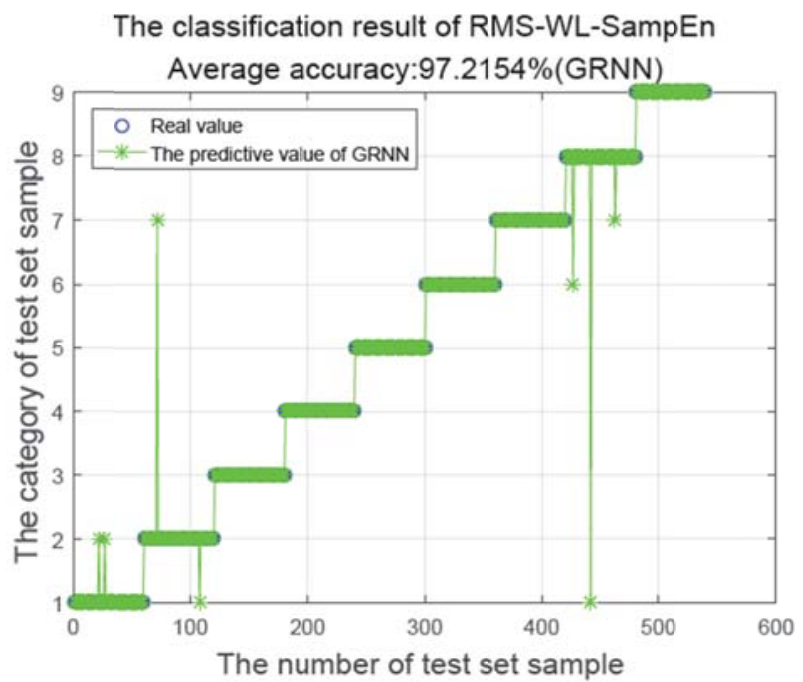

Fig. 8. The classification result of RMS-SampEn-WL.

As shown in Figure 6 and Figure 7, the classification rate of RMS is 86.5904\%, the classification rate of WL is $89.1204 \%$. The classification result of RMS-SampEn-WL is $97.2145 \%$. So the fusion feature is more excellent and reliable for hand motion pattern recognition.

\section{Conclusion}

In the paper, a multiple features fusion system for motion recognition is proposed. The feature, RMS-WL-SampEn, obtains the highest recognition rate by the GRNN classifier compared with two time feature (average $87 \%$ and $89 \%$ ). The new sEMG feature is effective and suitable for the sEMG pattern recognition. The results obtained from this experiment represent that the fusion feature can be a better way to increase the accuracy. The accuracy of the motion recognition was significantly improved to $97 \%$, which made the system more robust.

Although the feature extraction of static gestures is studied, there are still many problems to be further discussed. The feature dimension and redundant information can be reduced that will increase the speed of operation. Future research will use more subjects and more dynamic motions for data collection. Therefore, more efforts will be used in ensuring real-time processing for hand motion recognition. The selection of features need to be further studied, and the method used to reduce the dimension needs to be further determined. Finally, we hope to establish a real-time recognition system based on sEMG by optimizing the algorithm. 


\section{Acknowledgements}

This work was supported by Grants of National Natural Science Foundation of China (Grant Nos. 51575407, 51505349, 51575338, 51575412, 61733011), the Grants of National Defense Pre-Research Foundation of Wuhan University of Science and Technology (GF201705) and Open Fund of the Key Laboratory for Metallurgical Equipment and Control of Ministry of Education in Wuhan University of Science and Technology (2018B07).

\section{References}

1. Yang, W., Yang, D., Liu, Y., Liu, H.: A 3-DOF hemi-constrained wrist motion/force detection device for deploying simultaneous myoelectric control. Medical \& Biological Engineering \& Computing. 56 (9), 1669-1681 (2018)

2. Yang, D., Zhao, J., Gu, Y., Jiang, L., Liu, H.: Estimation of hand grasp force based on forearm surface EMG. In: 2009 International Conference on Mechatronics and Automation. pp. 1795-1799. IEEE, Changchun, China (2009)

3. Fang, Y., Zhou, D., Li, K., Ju, Z., Liu, H.: A force-driven granular model for EMG based grasp recognition. In: 2017 IEEE International Conference on Systems, Man, and Cybernetics (SMC). pp. 2939-2944. IEEE, Banff, AB (2017)

4. Yang, D., Zhao, J., Gu, Y., Jiang, L., Liu, H.: EMG pattern recognition and grasping force estimation: Improvement to the myocontrol of multi-DOF prosthetic hands. In: 2009 IEEE/RSJ International Conference on Intelligent Robots and Systems. pp. 516-521. IEEE, St. Louis, MO, USA (2009)

5. Li, G. F., Jiang, D. Zhou, Y. L., Jiang, G. Z., Kong. J. Y. Gunasekaran, M.: Human Lesion Detection Method Based on Image Information and Brain Signal. IEEE Access 7, 1153311542 (2019).

6. Sun, Y., Li, C. Q., Li G. F., Jiang, G. Z., Jiang, D., Liu. H. H., Zheng, Z. G., Shu, W. N.: Gesture Recognition Based on Kinect and sEMG Signal Fusion. Mobile Networks and Applications 23(4),797-805 (2018).

7. Jiang, X., Merhi, L.-K., Xiao, Z.G., Menon, C.: Exploration of Force Myography and surface Electromyography in hand gesture classification. Medical Engineering \& Physics. 41 63-73 (2017)

8. Koiva, R., Hilsenbeck, B., Castellini, C.: Evaluating subsampling strategies for sEMGbased prediction of voluntary muscle contractions. In: 2013 IEEE 13th International Conference on Rehabilitation Robotics (ICORR). pp. 1-7. IEEE, Seattle, WA, USA (2013)

9. Cheng, W. T., Sun, Y., Li, G. F., Jiang, G. Z., Liu, H. H.: Jointly network: a network based on CNN and RBM for gesture recognition. Neural Computing \& Applications 31(Supplement 1), 309-323 (2018).

10. Liao, Y., Sun, Y., Li, G., Kong, J., Jiang, G., Jiang, D., Cai, H., Ju, Z., Yu, H., Liu, H.: Simultaneous Calibration: A Joint Optimization Approach for Multiple Kinect and External Cameras. Sensors. 17 (7), 1491 (2017)

11. Nielsen, J.L.G., Holmgaard, S., Ning Jiang, Englehart, K.B., Farina, D., Parker, P.A.: Simultaneous and Proportional Force Estimation for Multifunction Myoelectric Prostheses 
Using Mirrored Bilateral Training. IEEE Transactions on Biomedical Engineering. 58 (3), 681-688 (2011)

12. Phinyomark, A., Scheme, E.: EMG Pattern Recognition in the Era of Big Data and Deep Learning. Big Data and Cognitive Computing. 2 (3), 21 (2018)

13. Sun, Y., Li, C., Li, G., Jiang, G., Jiang, D., Liu, H., Zheng, Z., Shu, W.: Gesture Recognition Based on Kinect and sEMG Signal Fusion. Mobile Networks and Applications. 23 (4), 797-805 (2018)

14. Triolo, R.J., Moskowitz, G.D.: The theoretical development of a multichannel time-series myoprocessor for simultaneous limb function detection and muscle force estimation. IEEE Transactions on Biomedical Engineering. 36 (10), 1004-1017 (1989)

15. Wu, J., Sun, L., Jafari, R.: A Wearable System for Recognizing American Sign Language in Real-Time Using IMU and Surface EMG Sensors. IEEE Journal of Biomedical and Health Informatics. 20 (5), 1281-1290 (2016) 